\title{
Circuit
}

Musiques contemporaines

\section{Chanter Dusapin : dialogues avec Françoise Kubler et Georg Nigl \\ Singing Dusapin: dialogues with Françoise Kubler et Georg Nigl}

\section{Vincent Ranallo}

Volume 29, numéro 1, 2019

Pascal Dusapin : la parallaxe des voix

URI : https://id.erudit.org/iderudit/1059425ar

DOI : https://doi.org/10.7202/1059425ar

Aller au sommaire du numéro

Éditeur(s)

Circuit, musiques contemporaines

ISSN

1183-1693 (imprimé)

1488-9692 (numérique)

Découvrir la revue

Citer cet article

Ranallo, V. (2019). Chanter Dusapin : dialogues avec Françoise Kubler et Georg Nigl. Circuit, 29(1), 25-38. https://doi.org/10.7202/1059425ar

\section{Résumé de l'article}

Les compositeurs et leurs interprètes collaborent à la création des oeuvres. La nature et le degré de cette collaboration sont variables. Cet article s'intéresse à la marque laissée sur la musique vocale de Pascal Dusapin par le contact des chanteurs Françoise Kubler et Georg Nigl. L'analyse se colore de l'expérience de l'auteur, lui-même praticien de la musique de ce compositeur. Le parcours du compositeur prend son essor dans une approche expérimentale, mise de l'avant dans la musique de chambre, et s'ouvre graduellement à l'opéra, oeuvre d'art total. Ce cheminement se cristallise dans les rapports de Dusapin avec Kubler et Nigl. À la question de l'expressivité de la diction, ou de sa neutralisation et de son abstraction, Dusapin répond par l'immédiateté de la dimension théâtrale, à travers les timbres de la voix et les mimiques corporelles. Le geste imaginé par le compositeur communique un affect. Il est non seulement vocal, mais il englobe le déploiement du corps chantant dans l'espace. 


\section{Chanter Dusapin : dialogues avec Françoise Kubler et Georg Nigl}

Vincent Ranallo

En interprétant en première nord-américaine O Mensch! Inventaire raisonné de quelques passions nietzschéennes (2008-2009), 27 pièces pour baryton et piano de Pascal Dusapin ${ }^{1}$, je suis entré en contact avec une œuvre d'une rare densité musicale et intellectuelle. Le compositeur lui-même est resté à distance de cette prise de contact, mis à part quelques courriels échangés où il me recommandait à son éditrice. Son écriture musicale, à la fois limpide et abrupte, évanescente et ciselée, se posait comme une roche sur laquelle mon collègue pianiste et moi avons aiguisé notre vigilance et limé notre endurance mentale. Ce grand cycle découle de la collaboration d'un compositeur avec un chanteur précis, Georg Nigl. Il s'agit d'une relation intime entre deux êtres. La grande difficulté de reprendre une œuvre si fortement marquée par la personnalité de son dédicataire attisait chez moi un besoin irrépressible de me démarquer. Assurer la première d'une pièce est comme pénétrer une terre inhabitée et créer soi-même les balises, sérieux incitatif à la vocation d'interprète créateur. Ces repères modifient la perception de l'œuvre lors de ses reprises subséquentes. La partition joue, dans ce processus, un rôle contradictoire : détacher l'œuvre de la personnalité d'un artiste unique et fixer une version «définitive», le document écrit ayant un effet contraignant sur l'esprit de l'interprète occidental'2.

Le degré de collaboration d'un compositeur avec ses premiers interprètes varie. Au-delà d'éventuelles connexions amicales ou amoureuses, les points de contact entre eux ressortent de la compétence instrumentale de l'un ainsi que du degré et de la nature de l'implication créative de l'autre. Dans ce texte, je propose de distinguer quatre modèles pour un tel partenariat, en déclinant les différents degrés de ces points de contact.
1. Dans le cadre de la résidence de Maxime McKinley à la Chapelle historique du Bon-Pasteur et de la saison régulière de la SMCQ, à Montréal, le 7 février 2014, avec le pianiste Matthieu Fortin.

2. Ce sujet mériterait un autre texte. Je ne soulève que le coin du voile. 
3. Benjamin Britten et Francis Poulenc, par exemple, tous deux compositeurs et pianistes, développent une connaissance intime de l'instrument de leurs partenaires respectifs, Peter Pears et Pierre Bernac.

4. Les vocalises de Quint dans The Turn of the Screw ont été façonnées par Britten sur le souvenir physique de la voix de Pears dans un organum de Pérotin. In Thy Beauty du compositeur québécois Marc Hyland trouve son origine dans une imitation ludique (par l'auteur de ce texte) de la voix de Kathleen Ferrier, contralto britannique. 5. Sakkas, 2010, p. 303-308

\author{
6. Instrument de musique \\ électronique, précurseur de l'actuel \\ synthétiseur. \\ 7. Colangelo, 1996, p. 48-50. \\ 8. En particulier, les Song Books. Voir \\ Charles, 1978, p. 145-150. \\ 9. Kim, 2018, p. 23-33.
}

1) Au début du XIX ${ }^{\mathrm{e}}$ siècle, plusieurs compositeurs sont réputés posséder une connaissance intime de la voix. Dans un contexte de production massive de nouveaux ouvrages lyriques, la grande proximité des chanteurs avec les créateurs et une certaine uniformisation de la morphologie vocale, suivant des principes esthétiques définis, semblent avoir favorisé cette compétence. Ce premier modèle est celui de la familiarité au fonctionnement de la voix. L'interprète n'intervient qu'au moment de la performance.

2) L'auteur conçoit une œuvre en fonction des moyens particuliers d'un interprète. Le timbre de sa voix, une approche musicale singulière, un engagement inspirant fournissent le point de départ d'une aventure créative souvent marquante et poussée 3 . Un chanteur peut frapper l'imagination du musicien par la qualité évocatrice d'une performance vocale publique ou privée ${ }^{4}$. Selon ce modèle, le compositeur connaît certains aspects du fonctionnement vocal et cette connaissance perçue, nourrie par un lien privilégié avec un interprète réceptif et engagé, soutient son inspiration.

3) L'interprète-chanteur joue le rôle de consultant en amont de l'acte compositionnel. Son partenaire musical possède moins de connaissances du fonctionnement normatif de la voix et le chanteur partage avec lui un éventail de possibilités incluant les registres, les timbres et les zones limites. Ainsi, le baryton grec Spyros Sakkas dévoile devant Iannis Xenakis toute l'étendue de son extrémisme vocal'5, tandis que Peter Maxwell Davies note les sonorités étranges (multiphoniques, cillements suraigus, etc.) que le comédien Roy Hart expérimente par la recherche théâtrale. Dans ce modèle, le chanteur fournit beaucoup d'information technique au compositeur, qui demeure malgré tout le créateur unique.

4) Les deux parties collaborent intensément dans un paradigme égalitaire où l'interprète est élevé au rang de cocréateur. Dans cette optique, le processus d'élaboration de Canti del Capricorno de Giacinto Scelsi met en jeu trois voix: le compositeur, le soprano Michiko Hirayama et un transcripteur. D'abord, une improvisation solitaire sur une ondioline ${ }^{6}$; ensuite, de la part du soprano, un travail d'arrimage avec les propriétés de sa voix; enfin, une transcription externe, révisée par la chanteuse et par Scelsi ${ }^{7}$. Des exemples supplémentaires seraient à trouver chez John Cage $^{8}$, Sandeep Bhagwati ${ }^{9}$ et plusieurs autres. Sur ce point, la question de la cosignature des œuvres cocréées pose un problème trop large pour être discuté ici. Crucial, il est résolu de manières diverses selon le tempérament des artistes, le compositeur conservant une sorte de priorité présomptive. 
Au fil des pages qui suivent, je m'intéresserai aux œuvres vocales récentes de Pascal Dusapin avec, en arrière-plan, cette idée de l'influence de certains chanteurs sur son écriture.

Dans les années 1970-1980, chez plusieurs créateurs cherchant à faire table rase des réflexes anciens, composer pour la voix force un renouvellement certain de la palette sonore. Le chanteur est un animal problématique: sa voix pose des questions. Elle véhicule une immédiateté de l'expressivité, ne serait-ce que par la présence du texte, élément hétérogène. On tient pour suspect l'opéra, symbole d'un conservatisme sclérosé. Lampleur même de l'aventure opératique crée un climat politique complexe. Dusapin lui-même, dès son premier opéra - Roméo G Juliette (1985-1988) -, connaît les luttes de pouvoir entre les différentes parties impliquées: bailleurs de fonds, librettistes, producteurs, diffuseurs, scénographes, metteur en scène, chanteurs ${ }^{10}$. L'écriture musicale et la vision unifiée du compositeur sur toutes les dimensions de son œuvre à faire sont relativisées. Ces commandes lyriques jouissent souvent d'une grande visibilitée ${ }^{11}$ tempérant la prise de risques esthétiques. Les exigences du chant scénique, projeté et intégré au jeu théâtral, limitent les modes non conventionnels d'émission vocale à moins d'avoir recours à l'amplification, pratique mal perçue par les praticiens du milieu.

À partir d'entrevues menées avec la soprano Françoise Kubler et le baryton Georg Nigl ${ }^{12}$, je tente de cerner l'adaptation de Dusapin à ces contraintes, alors qu'il fait le saut de la musique de chambre avec voix (depuis 1977) à l'opéra (1985 à aujourd'hui). Ces deux chanteurs marquent deux périodes de la production de Dusapin. Mon expérience professionnelle est également mise à contribution : les rapports que j'entretiens avec les compositeurs de la scène montréalaise créant des œuvres pour ma voix participent aux catégories décrites plus haut. Cependant, mon travail sur la musique de Dusapin laisse poindre un autre modèle: l'appropriation d'une œuvre conçue pour un autre artiste. Les enjeux de ce modèle sont complexes (l'adaptabilité, un niveau d'exigence différent, un possible sentiment de rivalité) et pourraient éventuellement servir de matière à un nouvel article.

\section{Connivences}

Pour Françoise Kubler (et pour son compagnon, le clarinettiste Armand Angster), la rencontre de Dusapin permet l'éclosion d'une camaraderie débordant la musique. Au début des années 1980, ils ont le même âge et commencent à s'établir dans le milieu. L'amitié naît d'emblée; le soprano décrit même une sorte de coup de foudre. La symbiose qui s'installe permet au duo, les membres fondateurs de l'ensemble Accroche Note, d'entrer dans un
10. Voir à ce sujet la conférence Un opéra en train de se faire sur le site du Collège de France (Dusapin, 2007).

11. Roméo \& Juliette (commande de l'Opéra de Montpellier, créé en 1989), Medeamaterial (La Monnaie, Bruxelles, 1992), To Be Sung (commande de l'ATEM, Nanterre, 1994), Perelà, Uomo di fumo (Opéra national de ParisBastille, 2001), Faustus, The Last Night (Staastoper Unter der Linden, Berlin, 2006), Passion (Festival d'Aixen-Provence, 2008), Penthesilea (La Monnaie, Bruxelles, 2015), Macbeth Underworld (La Monnaie, Bruxelles, sera créé en 2019).

12. Ces entrevues ont été réalisées par l'auteur via Skype en juin-juillet 2018. Toutes les citations de Kubler et Nigl dans la suite de ce texte sont tirées de ces entrevues. 
13. Gervasoni, 2013.

14. Pour voix de femme et piano.
15. Pour soprano, clarinette en la et
piano.

16. Il enregistra Bastien und Bastienne de Mozart en 1986 (Philips 420163-1).

17. Opéra qui sera créé à La Monnaie de Bruxelles en septembre 2019. véritable dialogue avec Dusapin. Ce dernier confirme la dette qu'il a envers eux: «Ils m'ont appris mon métier ${ }^{13}$.» La précision extrême de leur jeu trouve un écho dans le travail méticuleux du compositeur.

Françoise Kubler est une artiste du son, attachée à un déchiffrage et à une incarnation méticuleuse des idées notées par le compositeur dans une optique de musique de chambre. Elle jouit d'un intéressant point de comparaison puisque Dusapin retrouve l'égérie de ses premières armes après plusieurs années et lui offre Wolken ${ }^{14}$ (2014) et Beckett's Bones ${ }^{15}$ (2016). Ces deux œuvres témoignent d'une simplification et d'une complexification combinées que Kubler perçoit avec acuité.

La rencontre avec Georg Nigl a lieu vers 2005, alors que la notoriété de Dusapin atteint de nouveaux sommets. Les grands ensembles du monde et les hauts lieux de l'art lyrique le sollicitent. Les programmeurs de concerts reconnaissent à Dusapin le potentiel de toucher le mélomane. De son côté, le baryton connaît une carrière brillante et variée. Né à Vienne en 1972, cet ancien soprano du Wiener Sängerknaben ${ }^{16}$ possède une voix au timbre particulier, capable d'une grande vélocité et démontrant une large palette de nuances. Lorsqu'il est appelé à travailler avec Dusapin sur Faustus, The Last Night (2005), il jouit d'un statut de vedette. Le rapprochement entre le compositeur et sa nouvelle muse s'opère alors qu'ils se posent en égaux capables d'un dialogue qui dure encore aujourd'hui.

Nigl est un chanteur curieux, à l'affût des intentions du compositeur, le questionnant de vive voix et scrutant les détails de la partition. Dès le premier contact, il est clair que Dusapin est fasciné par cette voix dont il découvre les richesses inattendues. Un jour, Nigl chantonne à l'oreille du compositeur une canzonnetta de Monteverdi. Ce choc suffit à mettre en branle l'inspiration d'une nouvelle œuvre: dans Passion (2006-2007), le baryton fait large usage d'une ornementation puisée dans l'arsenal du proto bel canto, particulièrement d'un trémolo rapide et légèrement détaché, le trillo.

En composant Macbeth Underworld ${ }^{17}$, Dusapin parle au téléphone avec Nigl afin d'ajuster la partition aux moyens du baryton et de profiter de son expérience. Il achoppe sur la notation d'un passage particulier. Les signes, la tessiture et le phrasé utilisés ne sont pas bien accordés afin de transmettre l'affect escompté. Dans le dialogue, le compositeur précise son intention. Le chanteur, praticien habitué à saisir l'angle expressif le plus favorable à une performance convaincante, reconnait un archétype musical dont l'identification demeurait floue pour Dusapin. Ne s'agirait-il pas d'une berceuse? Une fois clairement nommé, le compositeur peut exprimer aisément la typologie de ce genre musical et ainsi trouver le ton juste. 


\section{Le texte par le corps}

Les deux chanteurs (Kubler et Nigl) abordent différemment le contenu verbal des œuvres. Le soprano possède une expérience marquée de l'improvisation. Ce contexte favorise la dimension du jeu sonore en minimisant la portée du signifié. Les œuvres composées pour Kubler entremêlent les couches textuelles, exploitant des modes de jeu étendus dans un contexte de «musique pure».

Pour le baryton, issu du monde de la musique baroque et du Lied autant que de l'opéra, le texte prime comme véhicule essentiel de communication. Formé à l'école de Nikolaus Harnoncourt - pour qui la rhétorique de la déclamation est maîtresse -, Nigl est avant tout un diseur. Les œuvres composées pour lui, dans toute leur modernité, peuvent se rattacher à la tradition classique. Le traitement agogique, la plasticité phraséologique et la sensibilité prosodique puisent directement à la source des œuvres de Claudio Monteverdi, dont l'ombre considérable porte jusqu'à la seconde École de Vienne. Nigl excelle d'ailleurs dans une forme très subtile de recitar cantando, apparenté au Sprechgesang expressionniste. Le chanteur précise ce terme qu'il considère comme mal compris, en le remettant en contexte et en le liant à la technique d'appropriation d'un texte par Johannes Brahms en prévision de la composition d'un Lied, telle que relatée par son élève Gustav Jenner (1865-1920). Il se lève le matin et lit le texte, le disant comme les acteurs allemands de cette époque au Burgtheater de Vienne, c'est-à-dire en déclamant sur un large ambitus. Puis il dort, puis il mange, puis il compose. Cette description souligne l'aspect expansif et exploratoire du processus prosodique où domine l'idée de représenter le texte dans ses aspects physiques et pulsionnels ${ }^{18}$.

Le phonéticien Iván Fónagy discute du fondement pulsionnel de la phonation. La voix trahit des intentions dépassant la parole au moyen de mimiques buccales et glottales. L'émotion manifeste un ensemble de gestes différentiels distordant le message primaire, neutre, par une déformation buccale ou faciale et des variations dans la fluidité glottique. D’autres éléments paralinguistiques entrent en jeu, comme la respiration et l'accentuation, afin de définir la portée expressive d'un énoncé ${ }^{19}$.

La musique vocale de Dusapin évolue vers une attention au véhicule paralinguistique. Roméo E Juliette (1985-1988) et Il-li-ko (1987) sont des exemples d'un premier style où la neutralité et l'égalité du débit reflètent un désir de distanciation poétique. Lors de la création de Roméo ๒ Juliette, Kubler est témoin de la grande complicité qui unit le compositeur avec son librettiste,
18. Information tirée des entrevues de l'auteur.

19. Fónagy, 1991, p. 109-111. 


\section{IL-LI-KO}

Pièce romantique pour Soprano seule

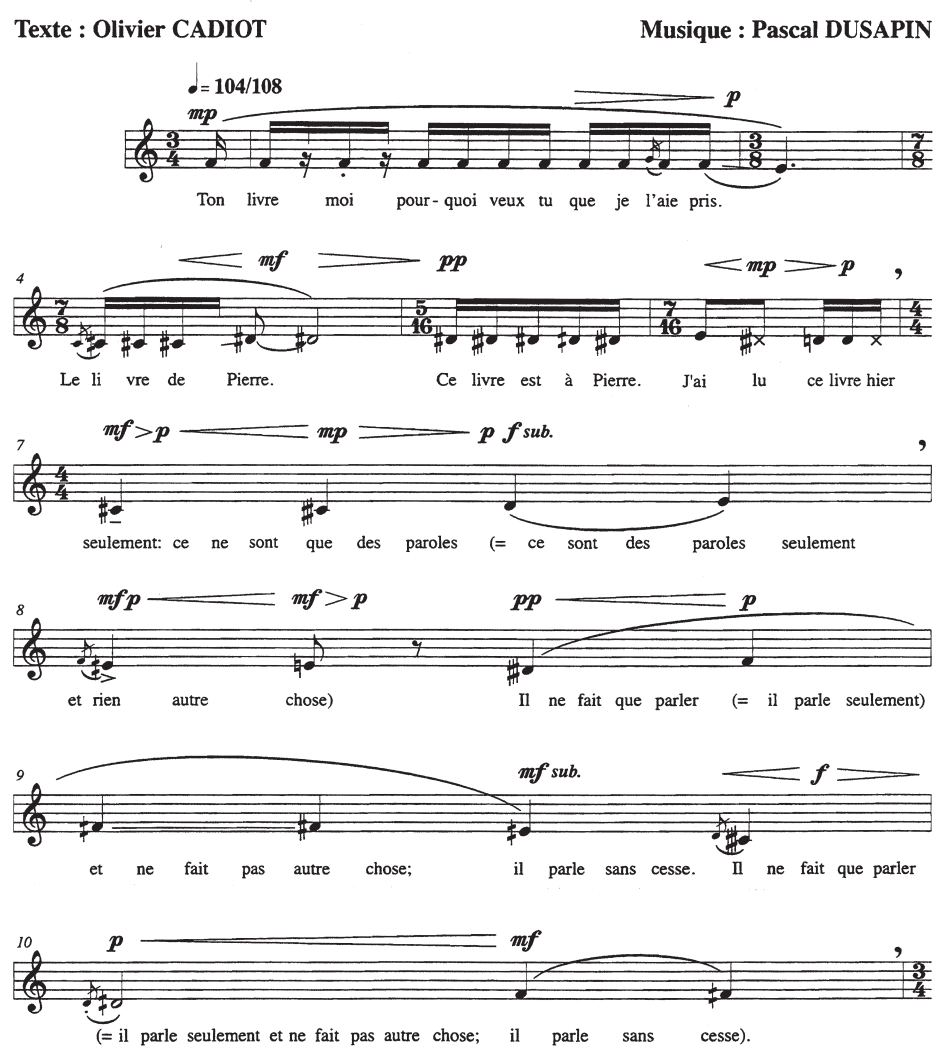

l'écrivain Olivier Cadiot. Les deux auteurs tentent de passer outre la dialectique texte-musique. Le style foisonnant du jeune compositeur rencontre le débit neutre, impersonnel et rapide qu'adopte Cadiot lorsqu'il lit ses textes. Cette manière réfractaire au lyrisme effusif transparaît dans leur opéra. Dans la pièce préparatoire Il-li-ko, pour soprano solo, Dusapin exploite une récitation précipitée et essoufflée, glissant par micro-intervalles. Il use de petites notes qui n'ont pas de finalité ornementale, mais accusent les resserrements du débit (Figure 1).

Lopposition conceptuelle décrite par Roland Barthes entre le géno-chant 
se base sur l'articulation précise de la langue, sur une diction distanciée presque structuraliste ${ }^{21}$; de l'autre, sur un geste vocal travaillé par la manière de dire, par le désir de représenter la vérité expressive, l'affect. Dusapin avec Cadiot s'imprègne d'abord du géno-chant. Dans sa musique instrumentale, il développe, selon l'analyse de Jacques Amblard ${ }^{22}$, des techniques inspirées de l'intonation de la voix. Dans sa musique vocale, il va plus loin en incorporant des signes paralinguistiques qui le rapprochent du phéno-chant.

Barthes, dans une réduction conceptualisante, oppose deux barytons célèbres de son époque: Charles Panzera pour le géno-chant et Dietrich FischerDieskau pour le phéno-chant. Lart de ce dernier est marqué par une extrême probité intellectuelle et par une attention maniaque aux détails de l'interprétation, visant l'expressivité. La rencontre artistique de Dusapin avec Nigl, héritier de Fischer-Dieskau, lui donne un partenaire de recherche inestimable. C'est un acteur doué. Dans son approche dramatique, il fouille sous l'écriture afin de faire surgir un personnage. Les œuvres écrites pour lui regorgent de notations d'articulation et d'émission pouvant se contredire ou relever de la gageure.

What I have to translate is what he writes down. But even in the moment he writes it down, it is already an interpretation. With Haas and Rihm, the composers try to write what the singer would do naturally, were it written more simply (portamento, rubato, timbre). But when you are on stage, you should not think like that, that makes it too complex. There is obviously the danger of forgetting all these indications from the start. I see myself as an advocate but with a big freedom.

Pour Georg Nigl, la dimension expérimentale pose un défi. Son statut de vedette internationale le mène à se produire dans le cadre des grandes maisons d'opéra européennes et des festivals internationaux de haut niveau. Dans ce contexte, la notion de risque constitue un enjeu crucial. Le droit à l'erreur est très restreint. Le baryton discute de la complexité que les compositeurs d'une certaine avant-garde recherchent souvent pour elle-même. Il a l'impression d'avoir à apprendre un nouveau langage à chaque nouvelle œuvre. Sa capacité à assimiler un style et à proposer une interprétation de premier plan dans des délais raisonnables guide ses choix artistiques. Cette attitude est une contrainte stimulante pour Dusapin. La difficulté de son langage trouve d'autres moyens de se manifester.

Lors de son premier contact avec Dusapin, les aspérités de la partition de Faustus, The Last Night poussent Nigl à travailler avec un professeur de solfège afin de s'assurer de la plus grande précision. Lexpérience d’un chanteur d'opéra diffère de celle d'un chanteur spécialisé en musique de chambre. Il doit mémoriser chaque détail, l'intégrer à son jeu théâtral et le projeter dans une grande salle, généralement sans amplification notable.
21. Le baryton suisse Pierre Mollet (1920-2007), quand il faisait travailler la mélodie française (Fauré, Debussy...), insistait sur la neutralité de la diction dont étaient exclus tous les éléments personnels, tous les épanchements emphatiques. II disait tenir cette approche d'Émile Vuillermoz (1878-1960), critique musical et compositeur, lui-même élève de Gabriel Fauré et ami de Maurice Ravel. (Souvenir de l'auteur.) 22. Amblard, 2002. 
23. Dusapin, 2007

24. Grâce à un dispositif réalisé par Thierry Coduys.

25. Constantin Stanislavski (1863-1938), Vsevolode Meyerhold (1874-1940). Tous deux sont théoriciens du théâtre et metteurs en scène russes. Voir PerelliContos, 1988.
Dans cette première collaboration, Dusapin cherche à rendre les frontières poreuses, entre chant pur, parlé-chanté, cri et chuchotement. Il vise une apparence d'improvisation bien que tout soit noté dans la partition. Le geste théâtral et vocal s'impose avec éloquence, apportant une grande force de conviction au discours musical du compositeur.

\section{Le chant dans le geste}

Bien que le cycle O Mensch! ne soit pas classé par l'éditeur parmi les opéras, Nigl et Dusapin mettent de l'avant l'aspect fortement scénique de cette pièce. Le baryton la qualifie d'opéra de valises. Un homme traverse une multitude d'affects contrastants, rappelant le Winterreise de Schubert. Toutefois, dans le chef-d'œuvre schubertien, la narration se coule dans la musique. Chez Dusapin, le «héros» revendique une présence extramusicale par le geste. Pour le compositeur, la gestuelle et la mise en espace prolongent l'acte compositionnel selon une logique graphique déjà présente dans des œuvres anciennes comme Roméo \& Juliette, où Dusapin imagine les dispositifs scéniques comme faisant partie de l'œuvre. Il les dessine et tente de les imposer au metteur en scène peu réceptif ${ }^{23}$. Le geste ne se limite pas seulement à un déploiement dans l'espace, il englobe les mouvements de l'émotion. Dans Passion (2006-2007), ces mouvements sont d'ailleurs captés directement par des électrodes et traités électroniquement ${ }^{24}$.

La corrélation entre geste et émotion, entre exploit physique et psychologie démarque les enseignements de Constantin Stanislavski et de Vsevolode Meyerhold ${ }^{25}$. Ce dernier développe une pédagogie particulière du théâtre, la biomécanique, qui se présente comme une gymnastique extérieure provoquant une réponse émotionnelle. Dusapin appelle une approche semblable. Dans la partition, il note des séquences articulées de durées, de hauteurs, de timbres (les voyelles, les caractères) et de dynamiques, séquences qui suscitent des gestes particuliers à poser dans une quasi-neutralité. Ces mouvements induisent des humeurs particulières que les spectateurs perçoivent et qui teintent la psyché du performeur.

Dans le septième tableau de Faustus, le personnage principal entre. Son chant commence sans assurance: les sons, dans le bas médium et le grave, mêlés de souffle alternent avec la voix parlée. À cet éventail de timbres fuyants et brouillés s'ajoutent des respirations purement rhétoriques, remplissant une fonction biomécanique. Elles plantent le germe d'un spasme nerveux. Faustus est hagard et désorienté. Il serait possible d'imaginer que le personnage retient sa respiration pendant les nombreux silences et qu'il suffoque soudainement. Dans le grave de la voix, les sons mêlés de souffle 


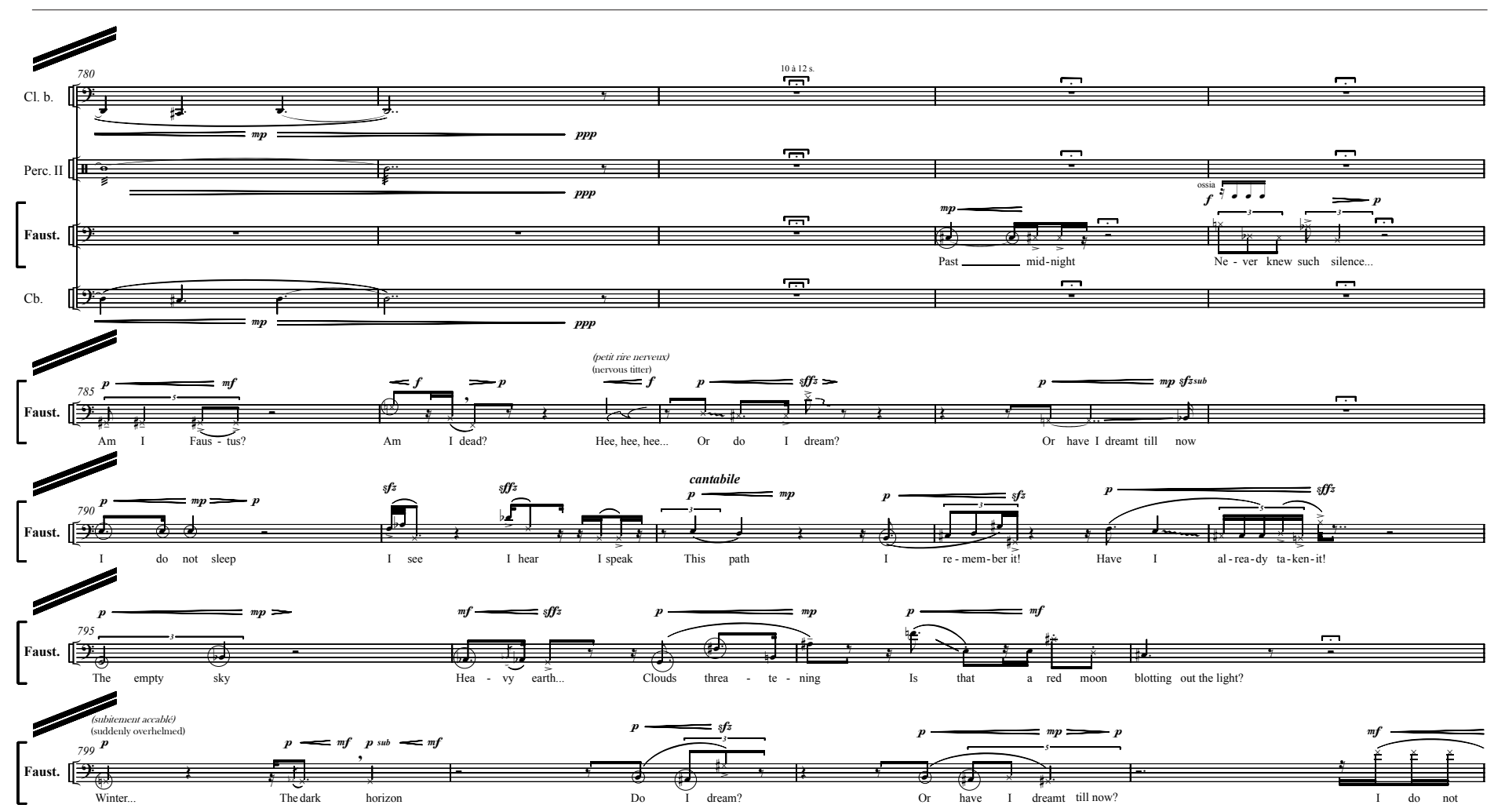

n’ont pas le même effet que dans le haut médium ou l'aigu: le résultat est assourdi. Le chanteur peut ressentir un état d'impuissance. En contraste, la suite de la scène exploite le registre aigu. La sonorité «i»sur He, avec glissandi ascendants, forme une vocalise, depuis le si grave jusqu'au sol aigu, qui puise dans une exclamation agressive. Le baryton britannique Thomas Hemsley associe à ce phonème exclamatif l'expression de l'hostilité, évoquant sa mimique grimaçante et la saturation de son spectre harmonique ${ }^{26}$. Ensuite, le phonème « 0 », associé à l'émoi, au trouble intérieur, colore l'invocation 26. Hemsley, 1998, p. 66-71. O Light. Le visage s'allonge, les yeux s'agrandissent. Ces gestes du faciès et de la bouche, l'effort articulatoire et les contraintes respiratoires, mouvements sous-entendus par la partition, mènent le chanteur vers la réalisation de l'acte musical et dramatique juste (Figure 2).

O Mensch! recèle également plusieurs passages dont l'efficacité expressive provient d'un fort ancrage mimétique. La partie centrale de Das Nachtlied ${ }^{27}$ 
déferle telle une danse sauvage, un geste endiablé. Le texte nietzschéen évoque une sorte de messie épuisé et méchant. Au piano, une série de claquements texturés par des acciaccaturas menaçantes pulse irrégulièrement le chant. Ce dernier, très sonore, s'appuie sur des accents emphatiques et évolue selon une métrique qui semble nier la cadence. Tous ces éléments contribuent à instaurer un sentiment de panique que le chanteur doit dominer afin de demeurer synchrone. Quatre invectives pleines d'orgueil permettent une projection plus frontale. Une sorte de sadisme s'installe ensuite, imagé par les appuis déphasés qui donnent presque à entendre un ricanement. Les points d'appuis de la ligne vocale se font de plus en plus ténus et capricieux jusqu'à se dérober et provoquer la chute, un aveu terrible souligné par un mouvement rythmique scandé, débouchant sur un cri dans le grave de la voix. Cette pièce déploie, à l'opposé de l'exemple précédent, un grand geste d'exaltation hargneuse et méprisante.

Das nächtliche Geheimnis ${ }^{28}$ relate un songe éveillé. Après une souple monodie posant le décor, la voix blanchit. Les interventions du piano se réduisent à des glas dispersés qui semblent trompeusement marquer des temps. Le texte s'arrime gauchement à la métrique et crée son propre espace dans une sorte de marmonnement. Le chanteur et le pianiste marchent dans le vide temporel. Compter pourrait se révéler hasardeux puisque la pulsation, endossée individuellement, risque une dissociation entre les deux partenaires. Il pourrait s'agir d'un malaise planifié par le compositeur, le texte traduisant une angoisse certaine, une suspension hallucinée.

\section{Le souffle dans la voix}

Françoise Kubler possède une voix aux caractéristiques fréquentes chez les chanteuses spécialisées en musique contemporaine. Le large ambitus comprend un registre grave exploitant la voix de poitrine de manière étendue. Le timbre de la voix est atypique et décomplexé. La rondeur n'étant plus la seule vertu, la rudesse gutturale et la nasalité s'émancipent de leurs fonctions expressives afin de mettre la voix à nu, débarrassée de ses affects et de son substrat culturel «bourgeois». La musique que Dusapin compose pour elle puise dans ces colorations et ce caractère. C'est elle qui fait découvrir au compositeur le contenu aérien de son timbre en s'inspirant de chanteuses de variété comme Jane Birkin.

Au début, on a beaucoup travaillé ensemble par rapport à l'écriture vocale. Je pense qu'il y a certaines choses que je lui ai proposées qu'il a trouvées intéressantes, comme la voix soufflée, qu'il n'utilisait pas à cette époque et qui est devenue courante chez lui. Il veut qu'il y ait du chant, la note chantée, mais avec du 
souffle, ce qui donne un son à la Jane Birkin. Tout le monde peut mettre un peu d'air sur la voix. Les chanteurs ont souvent des scrupules à le faire, mais là, c'est un affect presque théâtral. Donc on rajoute quelque chose de dramatique. Là où c'est le plus spectaculaire, c'est quand on arrête de le faire, quand on passe directement d'un son soufflé à un son normal. Parfois, cela nous échappe, on chante.

Certaines techniques, comme le son soufflé, s'adaptent mal à la projection. Nigl considère que ces sons posent un danger sur la tenue de sa voix. Les chanteurs honnissent le chuchotement voisé, assimilé à un serrement de la glotte qui ressemble à un raclement rauque. Le baryton les remplace parfois par des demi-teintes (ou mezza voce), couleur idiomatique dans la voix des artistes de sexe masculin. Cette dernière peut prendre plusieurs formes selon les esthétiques nationales. En Allemagne, les interprètes du Lied font appel à une coloration se rapprochant du falsetto, signe du relâchement de l'occlusion glottique qui laisse passer de l'air, conjugué à une tessiture tantôt élevée, tantôt grave, selon la texture recherchée. Toutefois, si le débit d'air expulsé devient excessif, la prise sur la voix tend à se désagréger et le chanteur risque le forçage. L'équilibre est donc fragile.

Chez Dusapin, les nombreux passages où les notes «emplies de souffle», «purement soufflées» et parlées s'entremêlent et se contaminent les unes les autres nécessitent un travail d'intégration théâtral. Dans la pratique, Nigl ne respecte pas tous ces signes à la lettre, mais en cherche la rhétorique sousjacente. En comparaison, mon approche se veut plus différenciée entre les sons soufflés, les chuchotements voisés, les sons parlés à voix basse. Au-delà d'une parole modulée, je crois qu'il y a recherche de la variation des timbres traduisant une sorte de schizophrénie.

Si Kubler rattache le souffle sur la voix à la manière de chanter des Jane Birkin et Vanessa Paradis, à la voix hypersexuée d'une féminité imaginée par l'industrie musicale, Dusapin se réfère plutôt aux habiletés des chanteurs de musique ancienne.

J'ai un grand amour pour Monteverdi et c'est en écoutant cette musique depuis si longtemps que j'ai pu appréhender ce type d'émission vocale qu'à la vérité, les chanteurs connaissent bien. Mais comme tous les chanteurs le savent, ce style est inséparable d'un lyrisme et des affects que suppose le texte. De ce qui est dit par la musique avec le texte ${ }^{29}$.

La voix devient comme une expiration. Le compositeur confie à Nigl un souvenir éloquent. Il raconte comment, au chevet de sa mère mourante, il épie cet air qui s'écoule, air qu'il sculpte en composant pour la voix ${ }^{30}$.

Cette manière de chanter est à mettre en rapport avec certains maniérismes du chant allemand. Plusieurs étoiles de l'Opéra de Vienne dans
29. Partition de $O$ Mensch!, notes du compositeur.

30. Information tirée des entrevues de l'auteur. 
les années 1960, comme Elizabeth Schwarzkopf, semblent parfois abuser d'un murmure aérien dans les passages de grande émotion des œuvres de Wolfgang Amadeus Mozart et de Richard Strauss. Cette technique est documentée dans l'histoire de l'art lyrique et se rattache au son de la voix parlée dans sa fragilité et sa plasticité.

Qu'il s'agisse de Birkin, de Schwarzkopf ou des interprètes de Monteverdi qui marquent Dusapin, ce jeu sur le timbre dont la forme extrême est l'atonie vocale tente de retrouver le naturel de la parole ou la texture de la voix non travaillée. Cette dernière représente même une sorte d'idéal pour certains compositeurs dont l'écriture s'accommode mal de la sonorité de la voix opératique conventionnelle, perçue comme lourdement connotée. Dans une entrevue autour de la création de son opéra Pastorale (2006), le compositeur Gérard Pesson évoque son désir de faire désapprendre à ses interprètes le «grand arroi lyrique ", à blanchir, à détimbrer la voix ${ }^{31}$. Conscient de l'intimité de ces techniques respiratoires et vibratoires avec le corps du chanteur, Pesson se réfère de la même manière aux spécialistes de la musique ancienne et baroque et au chant populaire et de variété. Au fond, ce grand arroi désigne le vibrato, grand suspect, et certains automatismes encombrants d'affects et de phrasés.

L'interprétation de la musique baroque propose une référence éloquente. Tout le travail des précurseurs, depuis Alfred Deller, Nikolaus Harnoncourt et William Christie, consiste à libérer l'interprète de ses automatismes afin de lui permettre l'acquisition d'un autre système rhétorique en phase avec la vérité historique.

Par ailleurs, la référence au chant populaire peut être liée à un instinct de décloisonnement: Kubler chante aussi du jazz, Cathy Berberian pose sur un même pied diverses approches stylistiques. Nigl cite en exemple Frank Sinatra et Miles Davis comme des exemples de complexité «instinctive» dans le phrasé et le timbre. La doxa actuelle tend à «démocratiser» l'art et à estomper les frontières des genres jusqu'à influencer le goût des artistes lyriques modernes. Lappréhension du timbre et du grain de la voix par le public et les pairs évolue. Ce qui aurait été inacceptable il y a cinquante ans, une émission droite et peu timbrée, est désormais approuvé et encouragé.

Kubler et Nigl s'entendent sur le fait que Dusapin encourage leur liberté d'interprète, qu'il demeure à l'écoute de la réalité du chant. Tous deux parlent du plaisir physique et intellectuel qu'apporte la performance de sa musique aux musiciens. 
Composant, Dusapin expérimente dans sa voix ${ }^{32}$. Par ses huit opéras, il se pose en collaborateur régulier des artistes lyriques. L'intensité et l'intimité de ces rapports lui permettent d’acquérir une compétence instrumentale qui le rattache au premier modèle proposé plus haut.

Le deuxième modèle s'applique aussi partiellement. Dusapin possède à l'origine peu de connaissances vocales. Il dit lui-même que l'opéra ne l'intéresse pas dans un premier temps ${ }^{33}$. Toutefois, le timbre particulier de $\mathrm{Nigl}$ et son approche de l'interprétation stimulent son approfondissement très personnel de l'écriture lyrique. La musique de chambre lui offre pour sa part un cadre expérimental, tandis que l'occasion de travailler sur des projets opératiques favorise l'expansion et le raffinement de son usage vocal en vue de communiquer des affects et de dépeindre des situations dramatiques.

Pour le chanteur, les approches de la musique de chambre et du théâtre lyrique commencent par s'opposer avant de se complémenter. La première se caractérise par un souci du détail et l'inclusion de toute expressivité dans le discours sonore, seul porteur de valeur. La seconde valorise les éléments paralinguistiques, le caractère rhétorique de la déclamation, la communication immédiate ou médiatisée de l'émotion par le geste.

Le désir de communiquer, de rejoindre le public, de lui transmettre quelque chose dévore Georg Nigl.

What does it all mean for people who don't know contemporary music? They are asking you. This is already a step. I asked this to my friend Helmut Lachenmann: "How would you answer this kind of question?" He said: "You hear two Japanese people talking to each other. You don't understand them. Then you try to start to listen. Suddenly you see that they are moving with their hands, their voice is going up and down, there are pauses, there is a reaction. Gradually, you get the feeling that you are getting involved, and in the end, you might have the feeling that you start to understand what they are talking about." This is a beautiful picture. Of course, we do music to touch other people.

Cette réponse donne le ton. Au-delà de ce qui est dit, les mouvements, les signaux visibles et audibles créent le sentiment de joindre la conversation. Ces gestes qui constituent la manière et qui suscitent l'adhésion, Dusapin en fait un élément essentiel de son langage et porte sur eux sa créativité. Composer l'indéfinissable. 


\section{B IB L I O G RAP H IE}

Amblard, Jacques (2002), Pascal Dusapin: l'intonation ou le secret, Paris, Musica ficta.

Barthes, Roland (1982), L'obvie et l'obtus: essais critiques III, Paris, Seuil.

Charles, Daniel (1978), Gloses sur John Cage, Paris, Union générale d'éditions.

Colangelo, William (1996), The Composer-performer Paradigm in Giacinto Scelsi's Solo Works, New York University, thèse de doctorat.

Dusapin, Pascal et Pouradier, Maud (2013), «Le corps en sous-bois », Nouvelle revue d'esthétique, $\mathrm{n}^{\circ} 12$, p. $143^{-154}$.

FónAgY, Iván (1991), La vive voix: essai de psycho-phonétique, Paris, Payot.

Gervasoni, Pierre (2013), «Accroche Note, ébouriffant», Le Monde, 17 septembre.

Hemsley, Thomas (1998), Singing and Imagination, Oxford, Oxford University Press.

KIM, Jin-Ah (2018), «Sur la comprovisation de Sandeep Bhagwati : théorie et pratique », Circuit, musiques contemporaines, vol. $28, \mathrm{n}^{\circ}$ 1, p. 23-33.

Perelli-Contos, Irène (1988), «Stanislavski et Meyerhold: pionniers de la pédagogie théâtrale», Études littéraires, vol. 20, nº 3, p. 13-25.

SAKKas, Spyros (2010), «Singing... Interpreting Xenakis”, in Sharon Kanach (dir.), Performing Xenakis, Hillsdale; New York, Pendragon Press, p. 303-334.

\section{ADRESSES URL}

Dusapin, Pascal (2007) «Un opéra en train de se faire», http://www.college-de-france.fr/site/ pascal-dusapin/course-2006-2007.htm (consulté le 22 novembre 2018).

Pesson, Gérard et Bergnach, Laurent (2009), «Gerard Pesson. Pastorale: portrait d'un compositeur autour d'une œuvre », Anaclase, www.anaclase.com/content/gérard-pesson-pastorale (consulté le 22 septembre 2018). 